\title{
Digitizing Data Management for Intraoperative Neuromonitoring
}

\author{
Chantal ZBINDEN ${ }^{\mathrm{a}, \mathrm{b}, 1}$, Moritz STRICKLER ${ }^{\mathrm{a}}$, Murat SARIYAR ${ }^{\mathrm{a}}$, \\ Thomas BÜRKLE ${ }^{\mathrm{a}}$ and Kathleen SEIDEL ${ }^{\mathrm{b}}$ \\ ${ }^{a}$ Bern University of Applied Sciences, Bern, Switzerland \\ ${ }^{\mathrm{b}}$ Department of Neurosurgery, Inselspital, University Hospital, Bern, Switzerland
}

\begin{abstract}
Intraoperative neurophysiological monitoring (IOM) enables a functionpreserving surgical strategy for surgeries of brain or spinal cord pathologies by neurophysiological measurements. However, the IOM data management at neurosurgical institutions are often either not digitized or inefficient in terms of collecting, storing and processing of IOM data. Here, we describe the development of a web application, called IOM-Manager, as a first step towards the complete digitization of the IOM workflow. The web application is used for structured protocoling based on standardized protocol entry catalog, data archiving, and data analysis. These functionalities are based on the results of the requirement engineering of a process analysis, a survey with potential users and a market analysis. A usability test with one IOM team indicated the IOM-Manager and its other components can in fact solve many problems of existing solutions.
\end{abstract}

Keywords. Intraoperative Neuromonitoring, Intraoperative Neurophysiology, Evoked Potentials, Brain Mapping, Data Management, Protocol Entries, Data Archiving, Data Analysis

\section{Introduction}

During surgery of infiltrative brain tumors, it is a challenge to distinguish healthy from pathological brain tissue. Even more important, functional boundaries of eloquent tissue (such as motor, sensory or language areas and tracts) are invisible to the surgeon. Such areas may be located directly next to the tumor or even be cancer infiltrated. If functional areas are injured during a tumor removal, postoperative deficits will occur, which worsen the patient's quality of life and therefore the prognosis. Postoperative deficits may be, for example, transient as well as permanent symptoms of motor paralysis [1-2].

Intraoperative neurophysiological monitoring (IOM) enables continuous measurements of the neuronal electrical activity during surgery, aiming to reduce the risk of postoperative deficits. IOM consists of methods for function localization (mapping) and continuous functional monitoring to assess the functional integrity. Through repetitive neurophysiological examinations, (e.g. evoked potentials), these techniques help to recognize an impending functional impairment during a neurosurgical operation and to prevent a possible deficit by adapting the surgical strategy [3].

${ }^{1}$ Corresponding author: Chantal Zbinden, Department of Neurosurgery, Inselspital, University Hospital Bern, Switzerland, chantal.zbinden@insel.ch. The authors claim that there are no conflicts of interest. 
The current IOM documentation workflow at the department of neurosurgery of the Inselspital IOM unit is mainly paper based and leads to redundant data, which increases the susceptibility to errors and makes it difficult to query the data. The goal of this project was therefore to digitize the entire IOM documentation workflow and to support different kinds of queries on the data, thereby fostering secondary use for research purposes. As part of this effort, the IOM-Manager has been implemented.

\section{Material and Methods}

Information acquisition with respect to the goal of our project involved a literature search on IOM documentation tools, a market analysis of medical devices in the area of IOM, a survey of potential users and the search for related systems. Pubmed and Google Scholar were consulted for the literature research using the following two search strings: (i) "Intraoperative Neuromonitoring" AND (reporting OR protocol OR documentation OR recording OR "data management") and (ii) "Intraoperative Neuromonitoring" AND (documentation OR "data management")". Information about IOM medical devices, potential users and similar systems were obtained online using specific search terms, by visiting a user meeting of a medical device manufacturer, and during the ISIN (International Society of Intraoperative Neurophysiology) congress in Vienna [4].

In order to understand the IOM workflow, in particular its documentation process, and to find ways to optimize it, the project team carried out a process analysis during three visits to the neurosurgery operating room. Process modeling was done in BPMN (Business Process Model and Notation) 2.0 [5]. The process analysis and interviews with the IOM team made it possible to capture central requirements and determine the functional scope of the application. Using systems analysis methods, covering data flow modeling, process analysis and variant analysis, we developed a concept for digitized IOM data management. This concept was the basis for a web application, called IOM-Manager, which was implemented with the MEVN technology stack. MEVN is an acronym and stands for MongoDB as a database, Vue.js as a frontend and the backend consisting of Express.js and Node.js. The advantage of using a MEVN stack is that one language is used throughout the whole application [6]. The IOM-Manager was evaluated by means of a usability test with the IOM team and compared to the existing paper-based workflow during a surgical intervention.

\section{Results}

\subsection{Process analysis results}

The IOM team consists of four medical technicians and a neurosurgeon/neurophysiologist. They document every case with a paper-based IOM protocol and keeps case statistics in a surgical track record (book) and an additional spreadsheet. An IOM protocol contains time stamped data of clinical events, e.g., loss of functions or relevant changes of the signals. Paper prints of the medical device signal curves containing the measured potentials during the intervention are added to these protocols. From the IOM device itself (inomed ISIS IOM System) individual IOM measurements could be exported as EDF files (European Data Format), which would enable digital evaluation [7]. These data are time series data. However, due to medical device regulation issues this feature 
is currently not integrated into the hospitals IT network. Thus, today the curves are still printed and analyzed by hand for specific research questions. The hospital collected more than 4000 protocols so far.

The IOM process itself comprises preoperative preparation, the monitoring process, including calibration of the baseline values related to the measurements, and postoperative procedures. The latter involves documentation, billing, archiving EDF files manually and different types of data analysis.

The following process weaknesses were identified: Converting the already digital information into analogue by printing out the signal curves. Double documentation with related information of the case statistics in a spreadsheet and in the surgical track record, making it difficult to find the IOM cases in the archive. No precise and efficient analysis is possible due to paper logs and curve printouts. The form and the quality of the protocol entries (free text) depend on the documenting IOM team member and finally the threemonth delay for data archiving.

\subsection{Results from literature research and market analysis}

The Pubmed search for "Intraoperative Neuromonitoring" produced 743 hits, which were further condensed down to 9 publications. Most of the publications were case descriptions and workflow protocols. These publications often describe the application and standardization of IOM in thyroid surgery. They contained no information about the actual protocol documentation of the data. Thus, no relevant search results for the design and implementation of the IOM-Manager were found.

Our survey involving $23 \mathrm{IOM}$ users lead to the following results related to the actual practice of IOM documentation: Eight of them used paper-based documentation. Four of them had a supporting IT system, which, however, would only cover some partial aspects of IOM documentation. The comment function of the IOM measurement machines itself was used in eleven institutions. Four of them used the comment function in combination with a paper-based documentation.

Market analysis and the survey demonstrated, that there is currently no system that fully meets the requirements of the Inselspital IOM unit and other potential users. The market analysis includes five medical IOM devices and related anesthesia information management systems. Especially, enhancing documentation is not a priority for many of the companies and they stated that they would not consider data management to be their job. This can be seen as an opportunity for the IOM-Manager to meet the needs of the target group in this area.

\subsection{Concept for a digitized IOM}

Figure 1 shows the components that are needed for a digitized IOM documentation. In this work some of these components (dark blue) have already been implemented.

1) The IOM measurement machine is a medical product of a commercial medical device manufacturer. It connects to the patient with stimulation and measurement electrodes and continuously monitors neuronal activity during surgery.

2) An interface between the IOM measurement machine and the IOM-Manager. Such an interface is required to access the measurement data. 
3) The IOM-Manager is a new web application implemented by us, which runs on a clinical workstation. The functionality of the application comprises digital case management and logging of IOM events during the surgery (see Section 3.4 for more details).

4) The common data pool has been realized for those parts required for the IOM-Manager. The current database model of the IOM-Manager supports data management for all IOM cases in a common database. Each case includes information about the patient, the operation and the protocol entries. The EDF files exported from the IOM measurement machine can be attached to the respective case.

5) Partial datasets for specific research questions could be extracted from the common data pool coded to support data analysis e.g. for a study cohort of patients with a given disease.

6) The BI (Business Intelligence) component for knowledge extraction has been implemented using the R programming language [8]. The JavaScript "R-script" library allows transfer of EDF files from the IOM-Manager to R and back. "R-script" enables data from the IOM-Manager to be transmitted to $\mathrm{R}$ via the Node.js server in JSON format, to execute the $\mathrm{R}$ functions and to import the results of the analysis into the IOM-Manager [9].

7) The results of the data analysis can be presented in different forms, such as processed and filtered signal curves.

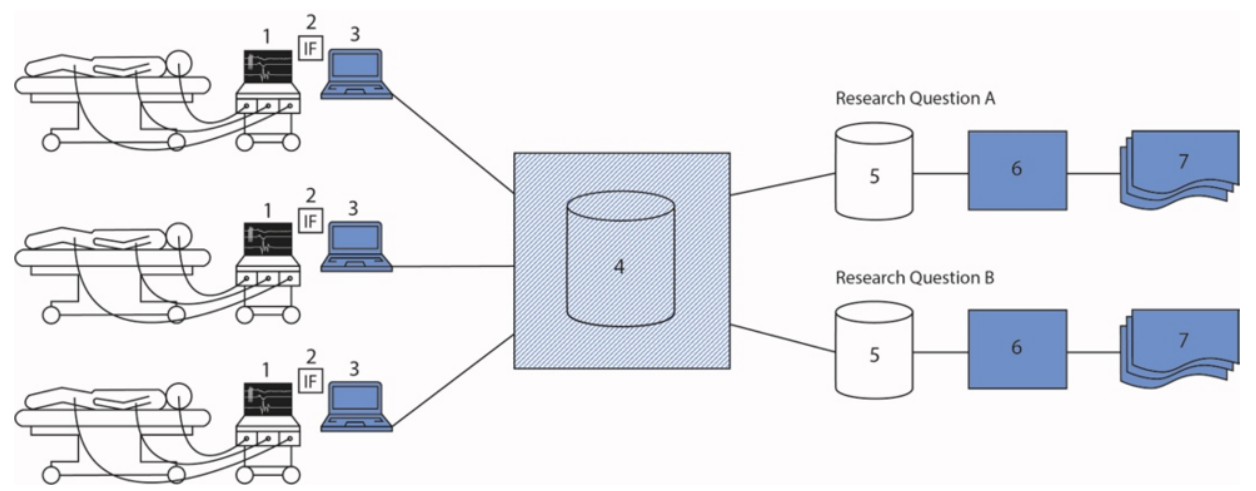

Figure 1. The graphic shows a concept for digitized IOM. In blue are the components that have already been realized. On the left side is an ongoing surgery of a patient who is connected with the IOM Measurement Machine. 1) IOM Measurement Machine, 2) Interface Machine-IOM-Manager, 3) Clinical Workstation running IOM-Manager, 4) Common Data Pool (partially realized), 5) Partial Data marts for certain Research Questions, 6) BI Component for Knowledge Extraction, 7) Analysis of the data

\subsection{IOM-Manager: Protocol entry catalog}

The IOM-Manager (component 3 of Figure 1, its data storage contributing to component 4 of Figure 1) is the central governing application for the digitized IOM data management. It supports management of all patients undergoing IOM and replaces the previous paper based IOM protocol (see 3.1). During each IOM procedure, it is essential to enable rapid data entry for each clinical event. Thus dropdown lists have been developed to select an event which is then stored with a time stamp in the IOM protocol of the respective patient (see Figure 2). In order to populate these lists, a complete protocol entry catalog was extracted from the latest 100 paper protocols. In this process, standardized formulations for the protocol entries were created. 
The catalog for the protocol entries is divided into four main categories: IOM, surgical procedure, anesthesia and others. Protocol entries are assigned to each category, which enables an improved evaluation of the clinical protocols. During the evaluation, it was found that the more than 200 protocol entries, 100 in one category, are too much to handle efficiently. Thus, the entries were further subdivided into 25 sub-categories [10].

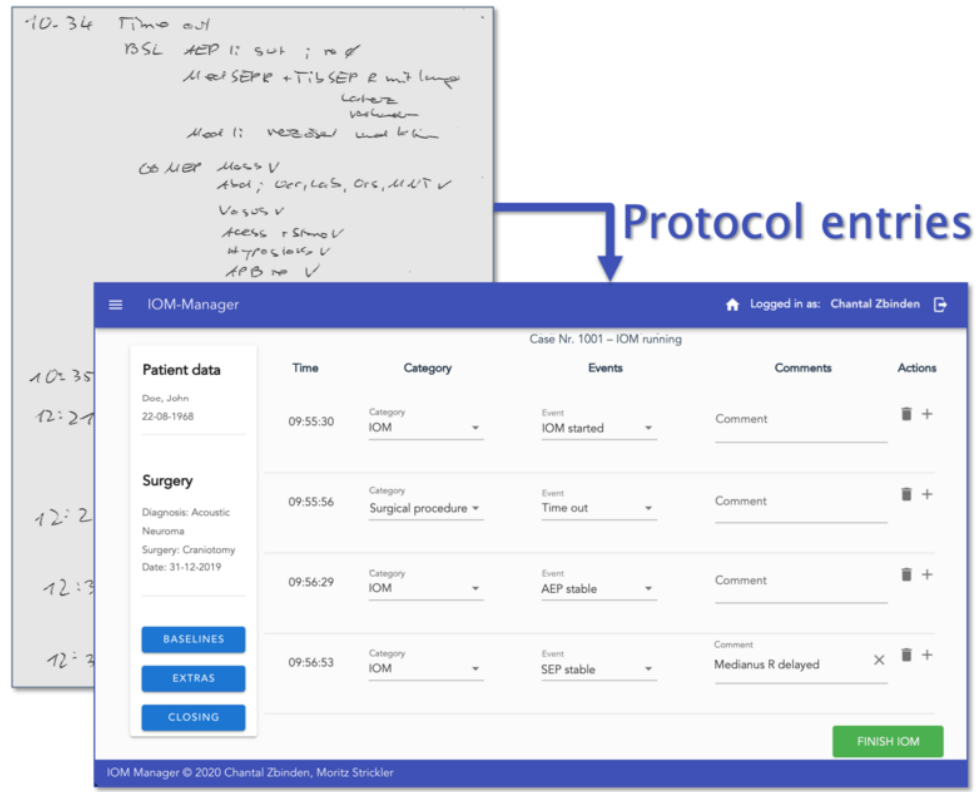

Figure 2. In the background, a section of a sample paper protocol of the Inselspital IOM unit can be seen. The foreground shows how the protocol documentation in our application looks like. On the left is information about the patient and the surgery. Each entry in the protocol contains a time stamp, a category and an event description. Furthermore, a free text comment can optionally be added to each entry.

\subsection{Evaluation results}

The IOM-Manger achieved a score of 94.5 out of 100 points in the SUS score (System Usability Scale), which corresponds to a very good usability rating [11].

\section{Discussion}

The IOM-Manager has the potential to fix most process weaknesses found in the Inselspital IOM unit. It received a consistently positive rating in the user evaluation and the comparative tests during interventions performed at the Inselspital IOM unit. Due to the exclusively digital documentation of the IOM cases, there is no longer any media discontinuity and no additional paper documents will be required. Duplicate documentation is eliminated, and the archived cases can be recovered easily. The standardization of the IOM protocols, together with the signal data uploaded to the IOM-Manager, enables better and more precise reuse of data.

Not all components of Figure 1 are implemented: the interface to the IOM measurement machine (component 2 of Figure 1) and the expansion of the common data pool (component 4 of Figure 1) are yet to be tackled. 
An interesting task for the future and goal of further efforts of our working group is the protocol entry catalog. First tests showed that more efforts may be required to reduce free text protocol entries. For future data analysis, it would also be advantageous if an entry could automatically be assigned to certain events in the protocol, e.g., by using and expanding the SNOMED CT nomenclature [12].

In addition to protocol events and signal measurement values, many other clinical data items may be relevant for assessment and research. Certainly, diagnoses and procedures must be considered as well as follow up results of clinical examinations of neurological deficits. Again, coded data would be preferable to uncoded data items and may allow us to find new clinical knowledge about lesion and resulting effects.

Thus, a variation of follow up projects are planned, e.g. the extension of existing functions such as structured protocoling and data analysis. The IOM-Manager will be tested in further tests by the IOM team in various surgical interventions and also in additional IOM centers.

\section{Conclusion}

The full digitization of IOM data management will improve documentation and will allow more effective and efficient data analysis. A first step has been realized with the development of the IOM-Manager and its other components.

\section{References}

[1] Schramm J, Boström A. Intraoperative neurophysiological monitoring - why we need it and a personal perspective of its development. In: Deletis V, Shils J, Sala F, Seidel K, Neurophysiology in Neurosurgery. $2^{\text {nd }}$ Edition. London, United Kingdom: Academic Press; 2020, xix.

[2] Chang EF, Clark A, Smith JS, Polley MY, Chang SM, Barbaro NM, Parsa AT, McDermott MW, Berger MS. Functional mapping-guided resection of low-grade gliomas in eloquent areas of the brain: improvement of long-term survival. Clinical article. Journal of Neurosurgy. 2011 Mar;114(3):566-73.

[3] Seidel K, Beck J, Stieglitz L, Schucht P, Raabe A. The warning-sign hierarchy between quantitative subcortical motor mapping and continuous motor evoked potential monitoring during resection of supratentorial brain tumors, Journal of Neurosurgy. 2013 Feb;118(2):287-96.

[4] ISIN 2019 Vienna $-7^{\text {th }}$ Congress of the International Society of Intraoperative Neurophysiology and Educational Curse [Internet]. ISIN 2019 Vienna. [cited 2020 Mar 25].

Available from: https://www.isin2019vienna.org/

[5] About the Business Process Model And Notation Specification Version 2.0 [Internet]. 2011 [cited 2020 Mar 30]. Available from: https://www.omg.org/spec/BPMN/2.0/About-BPMN

[6] Sharma A, Full-Stack Web Development with Vue.js and Node: Build scalable and powerful web apps with modern web stack: MongoDB, Vue, Node.js and Express. $1^{\text {st }}$ Edition. Packt Publishing. 2018.

[7] Kemp B, Olivan J, European data format 'plus' (EDF+), and EDF alike standard format for the exchange of physiological data, Clinical Neurophysiology. 2003 Sep;114(9):1755-61

[8] R: The R Project for Statistical Computing [Internet]. The R Project for Statistical Computing. [cited 2020 Mar 29]. Available from: https://www.r-project.org/

[9] r-script [Internet]. MIT. [cited 2020 Mar 29]. Available from: https://www.npmjs.com/package/r-script

[10] Zbinden C, Strickler M, Sariyar M, Bürkle T, Seidel K. A Protocol Entry Catalog for Intraoperative Neuromonitoring - Steps Towards an Ontology. Stud Health Technol Inform. 2020 Jun 26;272:31821.

[11] Brooke J, SUS - A quick and dirty usability scale. Usability Eval. 1996;(Ind. 189(194)):4-7. Available from: http://hell.meiert.org/core/pdf/sus.pdf

[12] SNOMED International. SNOMED CT [Internet]. SNOMED. [cited 2020 Mar 29]. Available from: http://www.snomed.org/ 